University of Wollongong

Research Online

Faculty of Engineering and Information

Faculty of Engineering and Information

Sciences - Papers: Part B

Sciences

2018

\title{
Modeling and Experimental Evaluation of Bending Behavior of Soft Pneumatic Actuators Made of Discrete Actuation Chambers
}

\author{
Gursel Alici \\ University of Wollongong, gursel@uow.edu.au \\ Taylor Canty \\ University of Wollongong \\ Rahim Mutlu \\ University of Wollongong, rmutlu@uow.edu.au \\ Weiping $\mathrm{Hu}$ \\ University of Wollongong,wh139@uowmail.edu.au \\ Vitor Sencadas \\ University of Wollongong, victors@uow.edu.au
}

Follow this and additional works at: https://ro.uow.edu.au/eispapers1

Part of the Engineering Commons, and the Science and Technology Studies Commons 


\title{
Modeling and Experimental Evaluation of Bending Behavior of Soft Pneumatic Actuators Made of Discrete Actuation Chambers
}

\begin{abstract}
In this article, we have established an analytical model to estimate the quasi-static bending displacement (i.e., angle) of the pneumatic actuators made of two different elastomeric silicones (Elastosil M4601 with a bulk modulus of elasticity of $262 \mathrm{kPa}$ and Translucent Soft silicone with a bulk modulus of elasticity of $48 \mathrm{kPa}$ - both experimentally determined) and of discrete chambers, partially separated from each other with a gap in between the chambers to increase the magnitude of their bending angle. The numerical bending angle results from the proposed gray-box model, and the corresponding experimental results match well that the model is accurate enough to predict the bending behavior of this class of pneumatic soft actuators. Further, by using the experimental bending angle results and blocking force results, the effective modulus of elasticity of the actuators is estimated from a blocking force model. The numerical and experimental results presented show that the bending angle and blocking force models are valid for this class of pneumatic actuators. Another contribution of this study is to incorporate a bistable flexible thin metal typified by a tape measure into the topology of the actuators to prevent the deflection of the actuators under their own weight when operating in the vertical plane.

\section{Disciplines}

Engineering | Science and Technology Studies

\section{Publication Details}

Alici, G., Canty, T., Mutlu, R., Hu, W. \& Sencadas, V. (2018). Modeling and Experimental Evaluation of Bending Behavior of Soft Pneumatic Actuators Made of Discrete Actuation Chambers. Soft Robotics, 5 (1), 24-35.
\end{abstract}




\title{
Modelling and Experimental Evaluation of Bending Behaviour of Soft Pneumatic Actuators Made of Discrete Actuation Chambers
}

\author{
Gursel Alici ${ }^{1,2}$, Taylor Canty ${ }^{1}$, Rahim Mutlu ${ }^{1,2}$, Weiping $\mathrm{Hu}^{1}$, and Vitor Sencadas ${ }^{1,2}$ \\ ${ }^{1}$ School of Mechanical, Materials, Mechatronic and Biomedical Engineering \\ ${ }^{2}$ ARC Center of Excellence for Electromaterials Science \\ University of Wollongong, 2522 NSW, Australia
}

Email: gursel@uow.edu.au

\begin{abstract}
In this paper, we have established an analytical model to estimate the quasi-static bending displacement (i.e. angle) of the pneumatic actuators made of two different elastomeric silicones (Elastosil MA 4061 with a bulk modulus of elasticity of $262 \mathrm{kPa}$ and Translucent soft silicone with a bulk modulus of elasticity of $48 \mathrm{kPa}$-both experimentally determined) and of discrete chambers, partially separated from each other with a gap in between the chambers in order to increase the magnitude of their bending angle. The numerical bending angle results from the proposed grey-box model and the corresponding experimental results match well that the model is accurate enough to predict the bending behaviour of this class of pneumatic soft actuators. Further, by using the experimental bending angle results and blocking force results, the effective modulus of elasticity of the actuators is estimated from a blocking force model. The numerical and experimental results presented show that the bending angle and blocking force models are valid for this class of pneumatic actuators. Another contribution of this study is to incorporate a bistable flexible thin metal typified by a tape measure into the topology of the actuators in order to prevent the deflection of the actuators under their own weight when operating in the vertical plane.
\end{abstract}

\section{Introduction}

As an emerging field of robotics, soft robotics is the science and engineering of the robots primarily made of soft materials, components and active structures such that they can safely interact and adapt with natural world better than their predecessors (i.e. robots made of hard components) [1-4]. Soft robots can be made of a number of rigid components or links connected to each other with single degree of freedom joints, like hyper-redundant manipulators or robots, whose each joint is controlled independently to realize a task or purpose [4-7]. However, this approach requires sophisticated algorithms to control not only the position of each link/component and whole robot, but also any interaction or contact between the robot and its physical environment. In line with recent progress in soft smart materials and additive manufacturing techniques, soft robots are expected to consist of a monolithic (i.e., continuum) structure containing actuation, sensing, motion/force transmission mechanism, energy storage (including batteries) units with a minimum foot-print to minimize the demand on sensory feedback and control. Such soft robots are expected to change their effective stiffness in order to provide a desired force when operating in various environments and interacting with various objects. When there is an application where a safe human-machine interaction and adaptability with the physical environment are required, there will be a need for a soft robotic system with variable and programmable stiffness. Another feature of soft robots is to incorporate the concept of morphological computation into their design [8,9]. The aim is to use the topology/morphology of the robot or its materials or its interaction with environment, or a combination of these to minimize the efficacy or function required from the controller. In short, the control functions are distributed over the morphology, materials, and constraints associated with a robot-environment interface. A typical example to morphological computation is to employ the concept of under-actuation to activate a multi-fingered gripper or a prosthetic hand [10]. 
Actuation is the most important feature to address when establishing soft robotic systems [1-2, 11-18]. Pneumatic and hydraulic actuators are favourable actuators to provide a high power density [18-24, 35-36]. Soft pneumatic actuators have a high power density, are lightweight, low cost, and provide the advantages of soft contact with a better morphing ability. They are also easy to manufacture using additive manufacturing and moulding techniques, which is why they have recently been the actuators of interest for soft robotic systems. Therefore, significant research efforts have been dedicated to establishing soft robotic fingers or hands or systems articulated with pneumatic actuators [11-16, 19-20]. The idea of using pneumatic networks consisting of small channels embedded in a slender body made of a hyper-elastic material such as silicone was reported by Wakimoto et al. [11] and Mosadegh et al. [13]. The channels can strategically be placed in a continuum body or in a discrete manner with gaps in between their exterior walls, removing the constraining effect of the continuum body, allowing large amplitude bending under the same input pressure. While former topology is named as slow pneumatic actuators, the latter is named as the faster actuators [13], due to the non-constraining chambers. The former will require much higher pressures than the latter to generate the same bending displacements. The chambers are designed in such a way that they allow a fast expansion in the longitudinal direction, with no discernible deformation in other directions, allowing a large bending of the slender body like a one-end fixed and the other end free cantilever beam. The performance of these actuators has been quantified experimentally and empirically using finite element modelling and analysis. For example, Wakimoto et al. [11] reported on the analysis, fabrication and performance characterisation of silicone rubber, discrete chamber actuators. A finite-element method (FEM) is used to undertake the analysis and optimisation of these actuators. Polygerinos et al. [12] have reported the establishment of quasi-static analytical and finite element models for fibre reinforced pneumatic bending actuators made of hyper-elastic silicones, not for chamber-based pneumatic actuators considered in this study. Hwang et al. [35] proposed pneumatic actuators consisting of trapezoidal vertical microballoon fins that were fabricated using $3 \mathrm{D}$ fabricated moulds. The performance of the actuators and their use in multi-finger grippers are comprehensively presented. Recently, Yap et al. [36] reported on employing a low cost printer based on fused deposition modelling (similar to the one used in this study to fabricate the moulds) using a soft commercial material called Ninjaflex (Shore Hardness 85A) to fabricate soft actuators with pneumatic chambers, which were previously fabricated using silicone based materials such as Ecoflex 030 and moulding techniques [11, 13]. This direct fabrication does not require supporting material, whose removal is a time consuming process, to construct the actuation chambers or networks. The primary issues associated with these actuators are that their durability is low, they require high actuation pressures, and their stiffness is constant. The high pressure and durability are coupled; the lower is the pressure, the higher is the durability. But, the lower is the pressure; the lower is the bending angle or displacement output and force output. Recently, Agarwal et al. [37] have proposed a new soft pneumatic actuator concept based on shell-reinforced patterns made of polyethylene terephthalate (PET) and a single air chamber made of a highly elastomeric material to allow bending and linear motions. The unstretchable shell enclosing the actuator chamber facilitates either bending motion or linear extension motion, depending on the pattern of the shell. Finite element analysis has been used to optimize the design parameters, and predict the performance and behaviour of the actuators. While they have employed the Ogden model to represent the nonlinear behaviour of the air chamber in the actuators, the linear elastic model is used to represent deformation of the shell.

There has been limited progress on establishing analytical models to better explain the phenomenon behind actuation and more importantly use these models better predict quasi-static and dynamic behaviour of the chamber-based actuators. With this in mind, we propose an effective analytical bending angle model and its experimental validation for this class of 
actuators. We also present a fabrication technique which requires fewer steps than the fabrication techniques reported before for the same type of actuators [11-13, 25, 34]. Another contribution of this study is to propose a bistable flexible thin metal typified by a tape measure to prevent deflection of the actuator under its own weight when operating in the vertical plan. This is a simple and effective way to keep the actuators in their straight configuration under no input. As the slender pneumatic actuators have a composite structure and nonlinear properties of the elastomeric materials used to construct the actuator, it is not straightforward to experimentally determine their modulus of elasticity using the classical technique of tensile tests. We have experimentally measured the bending angle versus pressure, and blocking force versus pressure data to identify the effective modulus of the actuators made of Elastosil MA 4061 with a bulk modulus of elasticity of $262 \mathrm{kPa}$ and a relatively soft Translucent soft silicone with a bulk modulus of elasticity of $48 \mathrm{kPa}$. We fabricated two actuators made of the same silicone; one with a constraining paper layer, and one with a bistable and flexible thin metal, in total 4 actuators have been experimented with in order to validate the analytical bending angle model.

In Section 2, we describe the soft pneumatic actuators and their fabrication, and experimentally determine the moduli of elasticity of the silicones they are fabricated from. Section 3 covers the derivation of the grey-box bending angle model, blocking force model and a simple kinematic model. The experimental results and validation of the models are presented in Section 4. The application of the proposed actuator to establish a soft robotic gripper consisting of 3 fingers and the use of the gripper in handling a regular object and an irregular object are discussed in Section 5. Conclusions and future work are presented in Section 6.

\section{Description and Fabrication of Soft Pneumatic Actuators}

The soft pneumatic actuator considered in this study is shown in Figure 1. It has a slender structure populated with discrete pneumatic chambers also known as pneumatic networks [1115]. Each chamber is separated into two sub-chambers by a rigid rib, which simplifies its fabrication compared to the fabrication of the previously published chamber-based pneumatic actuators based on moulding techniques. Another advantage of the ribs is that they prevent the expansion in the transverse directions. The chambers expand in the longitudinal direction of the actuator when activated pneumatically. While this is one way of adding structural strength to the actuator and preventing transverse deflection, the same constraining effect can be obtained by using fibre windings or shells as well-reported in the literature [12,13,20,21,37].
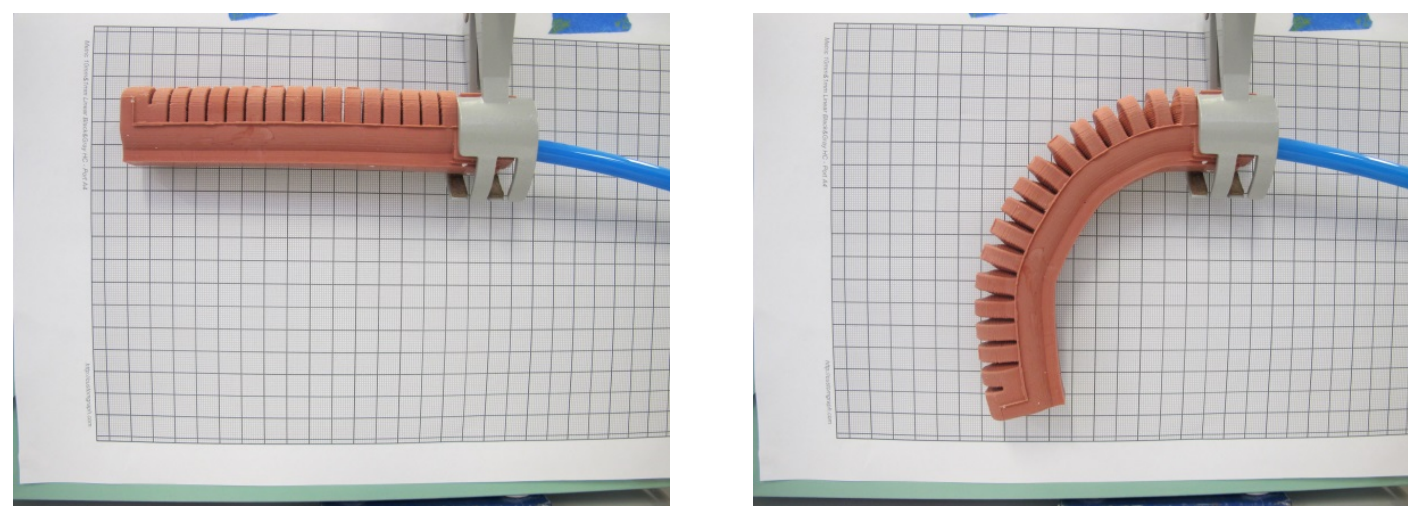


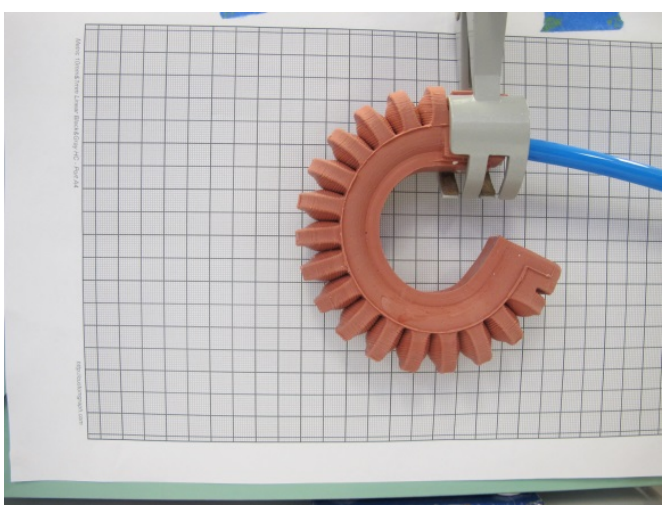

Figure 1: Configurations of the soft pneumatic actuator under various pressures: (a) $\mathrm{P}=0 \mathrm{kPa}$, (b) $\mathrm{P}=60 \mathrm{kPa}$, and (c) $\mathrm{P}=100 \mathrm{kPa}$.

As shown in Figure 1, the cantilevered actuator turns into a circular shape under a constant gauge pressure of $\mathrm{P}$. Its tip point generates deflections in the longitudinal and transverse directions. These deflections cannot be estimated using the classical beam theory, which is as accurate as $5 \%$ for the transverse beam deflections less than $20-30 \%$ of the beam length and assumes a negligibly small longitudinal deflection [26]. The soft pneumatic actuators made of hyperelastic materials generate large deflections, which introduce geometric nonlinearities and the materials nonlinearities in their mechanical output [20-24, 27, 37]. Therefore, there is a need for an effective mathematical model to estimate the bending displacements or the bending angle of the soft cantilevered actuator as a function of the input pressure [11-17, 28, 29]. Further, this model can be used to experimentally estimate the nonlinear parameters of the actuators such as effective modulus of elasticity.

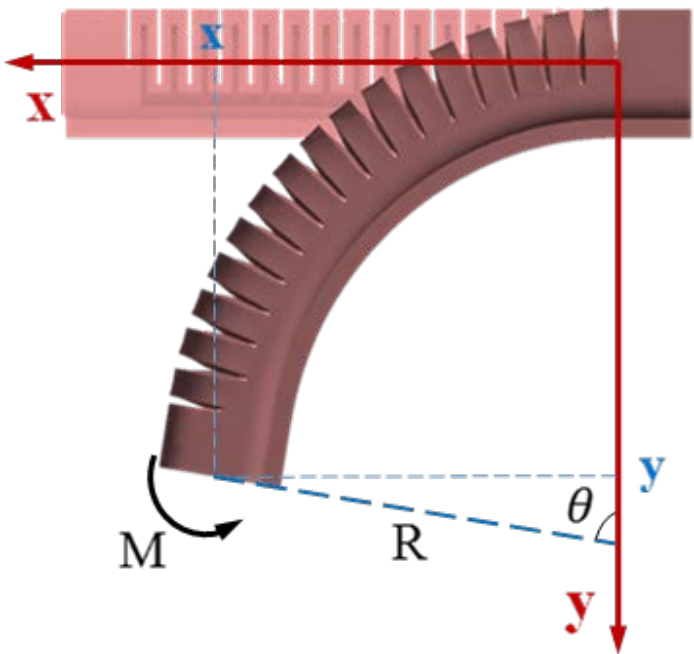

Figure 2: Deflection of a flexible beam (corresponding to the pneumatic actuators considered in this study) under an external moment $\mathrm{M}$ acting at its tip, resulting in a circular configuration.

\subsection{Fabrication of Actuators}

The actuator was fabricated using either of the two types of elastomeric silicones (Elastosil MA 4061, Barnes) and (Soft Translucent, Dalchem), and embedded with either a bi-stable layer (a thin flexible metal typified by a tape measure) or an inextensible layer (paper). In total, we fabricated and tested 4 actuators in this study. The actuators made of Elastosil MA 4061, and Soft Translucent silicone are simply called A1 and A2, respectively, for the sake of brevity in the rest of the paper. We used a low cost 3D printer to manufacture the mould that is comprised of three components. 
The fabrication process is designed so that an actuator can be moulded in one stage. This is an advantage over the similar actuators fabricated using a two stage process to create the chambers first and then close the chambers with a separately prepared layer obtaining a non-extensible layer such as a piece of paper or a piece of cloth or fibrous layer [13-16, 33]. As a first step, the inner mould was placed onto the lower mould with the spine jig in place. This step is to ensure the inner and outer moulds are oriented correctly. The silicone was combined with its polymerisation agent at a 9:1 and 10:1 weight ratio for the Elastosil MA 4061 and Soft Translucent silicones, respectively. The liquid silicone was thoroughly stirred not to fold air into the mixture. The mixture was then left to sit for approximately $5-10$ minutes to let some of the bubbles rise out. The liquid silicone was then poured into the assembled mould chambers in a long, thin stream to help prevent build-ups of air bubbles. The chambers of the outer mould were filled with just enough silicone such that it touched or engulfed the inner mould.

Using a vacuum desiccator, the mould and its contents were de-gassed. This expanded the mixture and caused most of the air bubbles to precipitate out. The silicone mould was cured in a chemical oven at $70^{\circ} \mathrm{C}$ for approximately 20-25 minutes. The upper mould was then clicked into the lower mould. The liquid silicone was then poured into the mould to just below the top of the upper mould, ensuring approximately $4-5 \mathrm{~mm}$ of space was left for the constraining material either a tape measure or a piece of paper. The mould and its contents were de-gassed and cured in the chemical oven at $70^{\circ} \mathrm{C}$ for another $20-25$ minutes. The bistable material (illustrated in Figure 3) or inextensible material should be cut to size and placed inside the top of the mould. The remainder of the liquid silicone was then poured into the assembled mould filling it to the top. Care was taken to ensure air was not trapped underneath the constraining material as this would affect its behaviour. Once more, the mould and its contents were de-gassed and then cured in the oven at $70^{\circ} \mathrm{C}$ for another $20-25$ minutes.

The actuator was then removed from the mould assembly. The 'ribs' were then snapped away from the inner mould by bending the chambers, with care taken to ensure all ribs were separated from the inner mould. Finally, the spine of the inner mould was removed using pliers, leaving the ribs in the chambers, as shown in Figure 3.

Soft robots are inherently soft in composition, however they are expected to perform tasks analogous to that of rigid robots and thus must, in some ways, behave rigidly. This issue was obvious during the design stage where the actuator would, at zero input pressure, bend under its own weight. Had the weight of the actuators been minimised such that there was negligible bending at zero pressure, there was still a fundamental lack of rigidity that would allow this actuator to be used in any practical scenario. To increase the actuator's rigidity by slightly increasing the input pressure, we propose an embedded bi-stable flexible thin metal in the form of a tape measure. Actuators embedded with an inextensible material such as a piece of paper, as suggested by Mosadegh et al [13], are restricted in one plane, turning any extension force into a bending moment. They are, however, still inherently prone to bending under their own weight and do not have sufficient rigidity such as that of the human spine or fingers.

By embedding the actuator with a bi-stable material such as a strip of tape measure as pictured in Figure 3, the actuator would behave stiff under zero input conditions and at a reasonably small input pressure would 'kick' into its second state of mechanical stability, allowing bending. Such a material would also be inextensible preventing lateral extension, analogous to the fibrous layer, simultaneously undertaking two functions [11-14, 20, 21]. 


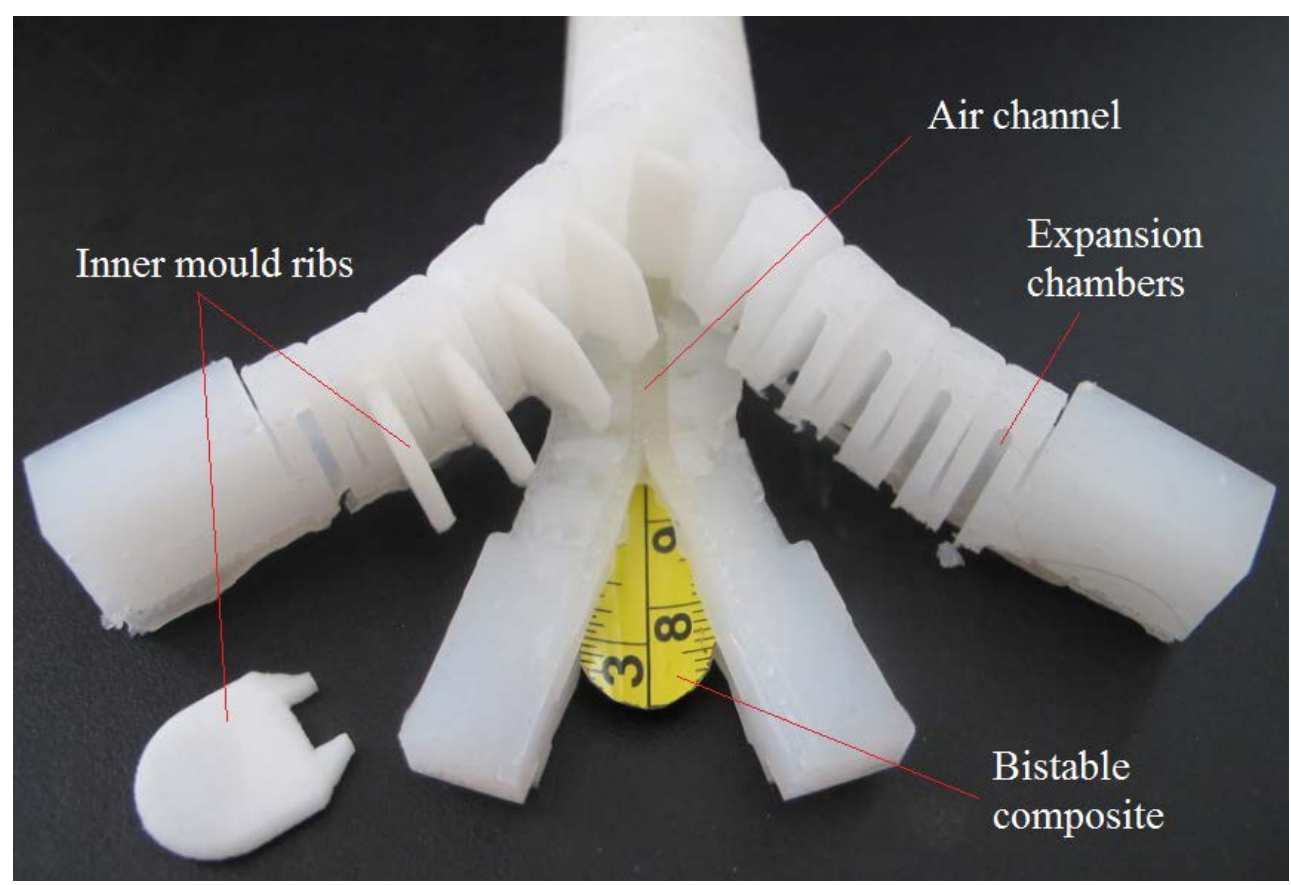

Figure 3: Dissection of the actuator made of translucent silicone with a bistable component that is a strip of a tape measure.

\subsection{Determination of Modulus of Elasticity}

The elastic modulus of the Elastosil M4061 and the Soft Translucent silicones, prepared like described in subsection 2.1, were determined using tensile tests (Instron tensile machine). Five samples were produced for each elastomer in order to find the average modulus of elasticity. The test pieces were manufactured using a dog-bone stencil cutter with the dimensions of $10 \mathrm{~mm}$ in length, $2 \mathrm{~mm}$ in width. The thicknesses of Elastosil silicone and soft translucent silicone were $115 \mu \mathrm{m}$ and $900 \mu \mathrm{m}$, respectively. For hyper-elastic materials, the modulus of elasticity is not constant as the stress-strain relationship is nonlinear. This is because the crosslinks between the polymer chains undergo realignment when induced with a strain. Once all of the cross-links have aligned with their respective polymer chains, the sample should fail shortly thereafter. Therefore, we have used the relationship between nominal stress $\sigma_{n}$, the shear strain between the polymer chains and shear modulus of elasticity, which is given by [30]

$$
\sigma_{n}=G\left(\lambda-\lambda^{-2}\right)
$$

where $\lambda$ is the extension ratio given by $\lambda=1+\varepsilon$, and $\varepsilon$ is the strain. The modulus of elasticity $E$ for elastomers with a large-strain elasticity is $E=3 G$. It must be noted that Eq. 1 is valid under assumptions of (i) the silicones are isotropic in all directions, and (ii) they are incompressible [30]. The experimental results from both silicones and corresponding average modulus of elasticity from these tests are shown in Figure 4 and Table 1, respectively.

Table 1: Modulus of elasticity results for both silicones from the tensile tests.

\begin{tabular}{|l|l|l|}
\hline Tests & $\begin{array}{l}\text { Elastic modulus E at 100\% shear } \\
\text { strain, Elastosil M4061 silicone, kPa }\end{array}$ & $\begin{array}{l}\text { Elastic modulus E at 100\% shear } \\
\text { strain, Soft Translucent silicone, kPa }\end{array}$ \\
\hline 1 & 203.1 & 61.5 \\
\hline 2 & 299.1 & 39.6 \\
\hline 3 & 271.8 & 41.7 \\
\hline 4 & 221.7 & 55.5 \\
\hline 5 & 316.2 & 41.7 \\
\hline Average & $262.4 \pm 48.7$ & $48.0 \pm 9.9$ \\
\hline
\end{tabular}




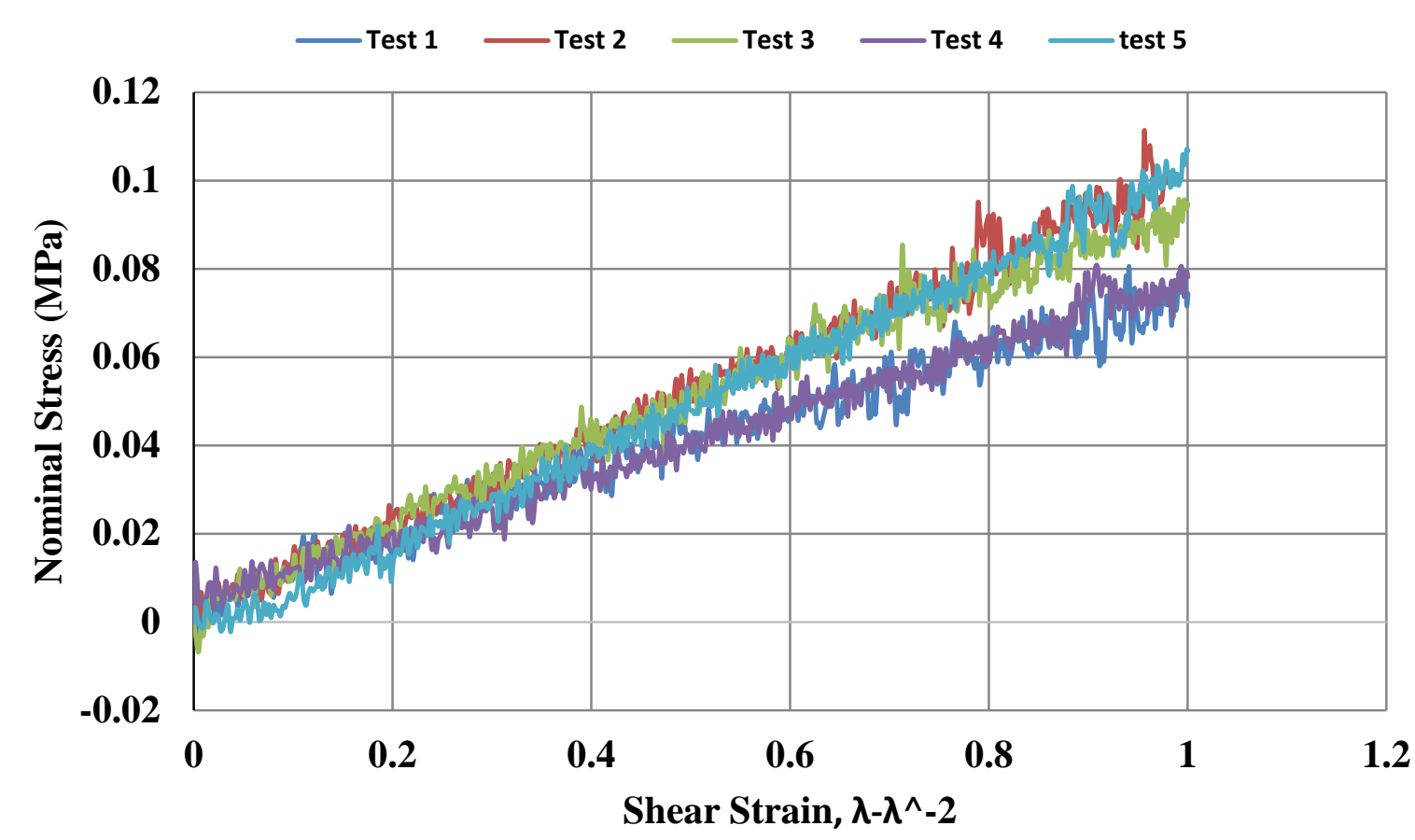

— Test 1 - Test $2-$ Test $3 \longrightarrow$ Test 4 Test 5

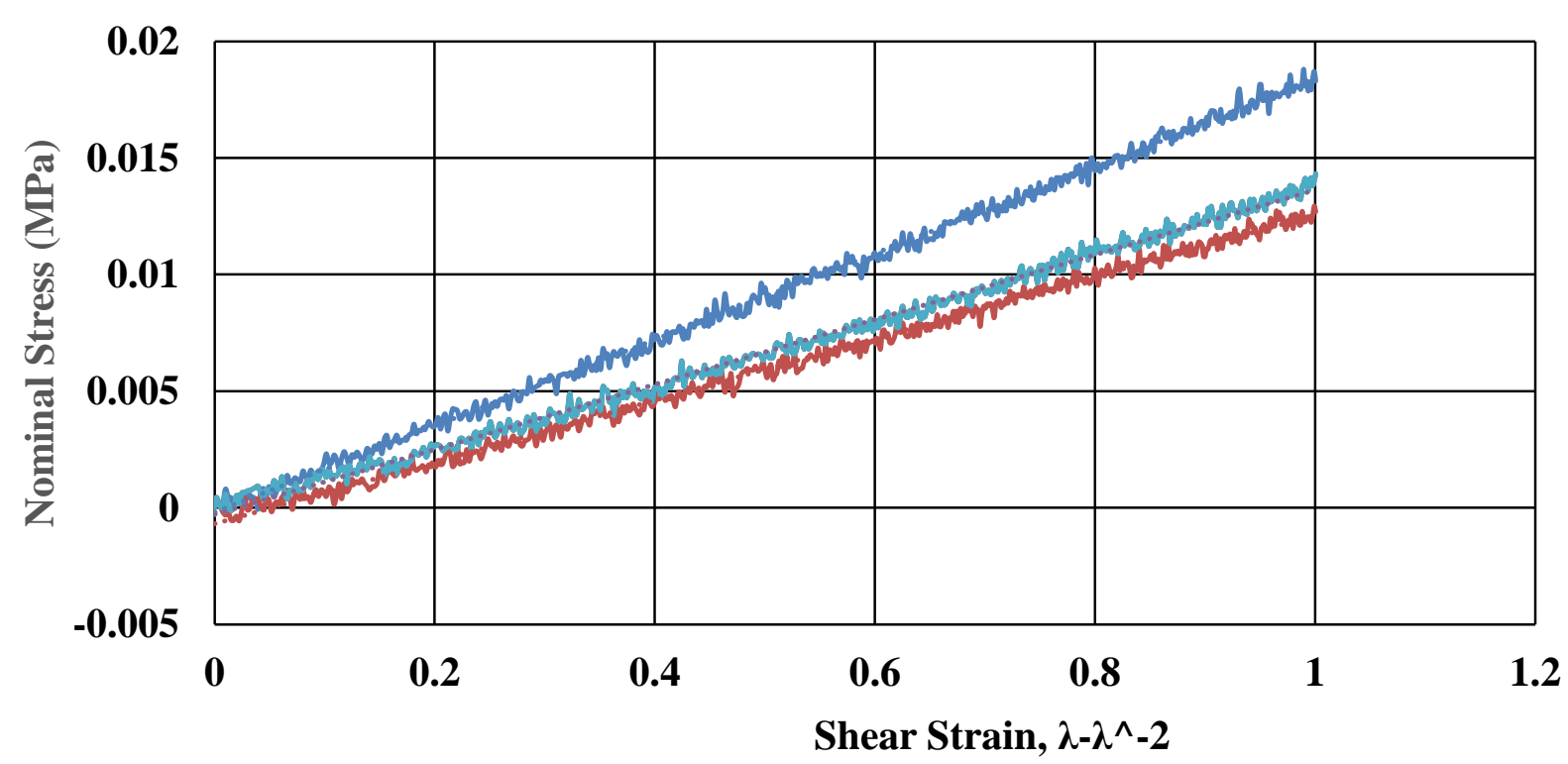

Figure 4: Nominal stress versus shear strain data (from 5 tests) to estimate the elastic modulus for (a) Elastosil silicone rubber (top), and (b) for soft translucent silicone (bottom). 


\section{Derivation of Bending Angle Model}

The material used to make A1 and A2 is an elastomeric material with a non-linear stress-strain curve. Further, the deflection of the actuators is nonlinear, which cannot be estimated using the classical beam theory. This follows that there are two sources of nonlinearities (material and geometric nonlinearity). The material nonlinearity is especially significant under compression stresses, as reported in [11,13]. In this paper, we assume that the material is under a tensile stress, which has a linear relation with the resulting strain for strains of up to $70 \%$ (Figure 3 of [12]). When the actuators are in action, they are in the form of a cantilevered beam. Using Euler-Bernoulli principle [26, 38], the radius of curvature of the actuator configuration in Figure 2 is given by

$$
\frac{1}{R}=\frac{M}{E I}
$$

where R, E and I are the radius of the curvature, modulus of elasticity and area moment of inertia of the deflected beam or actuator, respectively. Assuming (as illustrated in Figure 1) that it bends into a constant curvature shape, the corresponding bending angle $\Theta$ is simply

$$
\theta=\frac{L}{R}
$$

When the pressure $\mathrm{P}$ is applied to the actuator through the chambers distributed along the actuator length, it will apply a tensile force on the inner surface of the chamber, given by

$$
F=P A
$$

If the cross-sectional area of the chamber is regular like a circle or a sphere or a rectangle, the neutral axis will pass through centre of pressure, and the actuator will expand uniformly in all directions, with no bending. On the other hand, if there is a slight offset between the centre of pressure and the neutral axis of the actuator, the actuator will bend towards the side where the neutral axis is. The moment causing this bending is simply the tensile force F multiplied by the offset ' $e$ ' between the centre of pressure and the neutral axis, as shown in Figure 5a. The tensile force and the corresponding bending moment acts on the cross-section at the neutral axis, as illustrated in Figure 5a. The force acting on this cross-section is equal to the tensile force acting on the inner surface of the chamber

$$
P A=\sigma_{t} A_{w}=>\sigma_{t}=P \frac{A}{A_{w}}
$$

This stress $\sigma_{t}$ should be less than the tensile strength of the materials (which is $6.5 \mathrm{MPa}$ for Elastosil M 4601) from which the chambers are fabricated. 

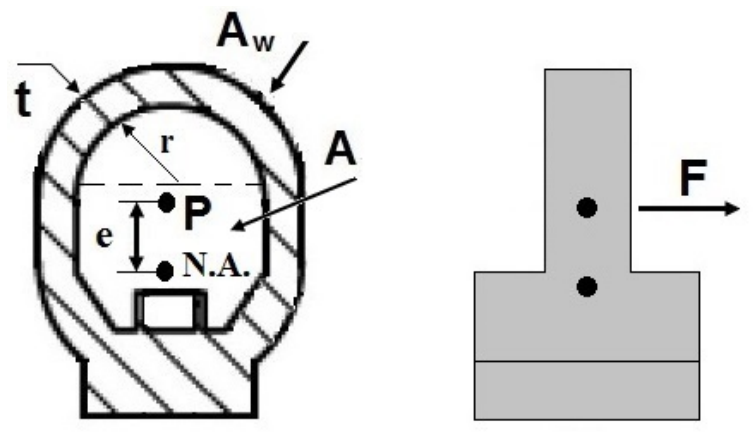

(a)
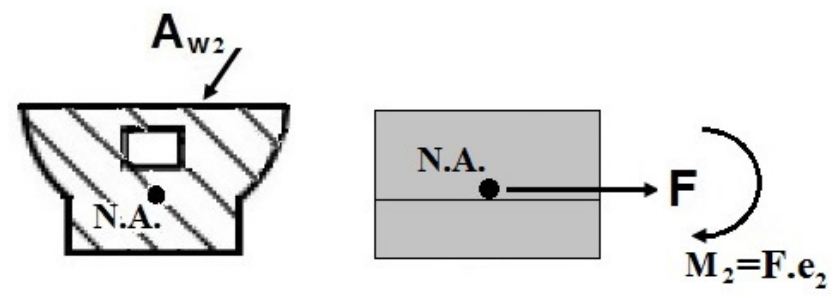

(b)

Figure 5: (a) Cross-section of each chamber and the resulting geometry under an internal pressure of $\mathrm{P}$, the force $\mathrm{F}$ acting (Eq.4) at the center of the chamber, and this force is transferred to the neutral axis of the cross-section with a bending moment $\mathrm{M}$, and (b) cross-section outside or between two chambers. It must be noted that the area of this

cross-section $A_{w 2}$ is much smaller than the cross-section $A_{w}$ in (a) and is further from the centre of pressure allowing more bending of the actuator.

Under the tensile force $\mathrm{F}$ (Figure 5a), the length of the chamber or the actuator segment will change by $\delta L_{i}$, which is given by

$$
\delta L_{i}=\frac{F L_{i}}{A_{w} E}=\frac{P A L_{i}}{A_{w} E}
$$

Similarly, under the bending moment (Figure 5a) $M=P A e$, the radius of curvature is given by

$$
\frac{1}{R}=\frac{P A e}{E I}
$$


When the actuator bends to a steady-state configuration like in Figure 1 or Figure 2, the new length of the actuator is

$$
L=L_{i}+\delta L_{i}
$$

Substituting Eqs. 6-8 into Eq.3 results in an analytical expression for the steady-state bending angle of the actuator;

$$
\theta(P)=\frac{P A e}{E I}\left(L_{i}+\delta L\right)=\frac{P A e}{E I}\left(L_{i}+\frac{P A L_{i}}{A_{w} E}\right)=\underbrace{\frac{L_{i} A^{2} e}{A_{w} E^{2} I}}_{C} P^{2}+\underbrace{\frac{L_{i} A e}{E I}}_{D} P=C P^{2}+D P
$$

This follows that the bending angle is a nonlinear function of the bending pressure, assuming that the other parameters in Eq. 9 do not change with the bending of the actuator. It must be noted that the second-order term $\left(P^{2}\right)$ is inversely proportional to the square of the modulus of elasticity E. For an actuator made of a relatively stiff material, this term can be disregarded and the actuator will show linear bending angle versus-pressure behaviour. It must be noted this model considers the chamber and gap between two chambers as a single unit with a length of $L_{i}$.

We have derived Eq. 9, by assuming that (i) the actuator cross-sections are perpendicular to the neutral axis during the deformation or bending, (ii) any deformation of in the chambers and cross-sections in the transverse directions are negligibly small, and the modulus of elasticity of the actuator is constant.

It must be noted this analytical model in Eq.9 can be extended to the slender actuators with no external gaps between the chambers - the actuator is a slender soft structure with an offset between its centre of pressure and neutral axis. When there is a gap or space between the external walls of two consecutive chambers, there will be two cross-sectional areas bearing the bending moment $\mathrm{M}$ and axial force $\mathrm{F}$ as shown in Figures $5 \mathrm{a}$ and $5 \mathrm{~b}$, respectively. With this in mind, the total angular deflection of the actuator, which is the sum of the deflections in these two cross-sections, is obtained by applying Eq. 9 to two cross-sectional areas of $A_{w}$ and $A_{w 2}$. When the gap between the chambers is widened, the length of the cross-section $A_{w 2}$ increases, which results in a higher strain in this cross-section and subsequently a larger overall angular deflection, as presented in Section 4.

\subsection{Blocking Force Model}

We drive an analytical force model based on the volume change in the chambers under a constant pressure. When a constant gauge pressure $\mathrm{P}$ is applied to the actuator, the chambers primarily expand in the longitudinal direction, forming a convex shape, as shown in the actuator in the bottom image of Figure 1. For a volume change $d V$ in the actuator under the pressure $\mathrm{P}$, the input work $d W_{\text {in }}$ causing the bending of the actuator is;

$$
d W_{\text {in }}=P d V
$$

For a cantilevered actuator free to bend under the pressure, the work output $d W_{\text {out }}$ can be related to the resultant bending moment $\mathrm{M}$ acting at the tip of the actuator and the corresponding bending angle $d \theta$;

$$
d W_{\text {out }}=M d \theta
$$


When the actuator is in the blocked force configuration, the bending moment is equal to the blocked $F_{B}$ times the length $L$ of the actuator. Using the definition of mechanical efficiency $\eta$, the work output is given by

$$
d W_{\text {out }}=\eta d W_{\text {in }}
$$

Substituting Eqs.10 and 11 into Eq. 12 results in

$$
F_{B} L d \theta=\eta P d V=>F_{B}=\eta \frac{d V}{d \theta}\left(\frac{P}{L}\right)
$$

Eq. 13 can be written in terms of the volume change and bending angle change per unit time as

$$
F_{B}=\eta \frac{\frac{d V}{d t}}{\frac{d \theta}{d t}}\left(\frac{P}{L}\right)=\eta \frac{\dot{V}}{\dot{\theta}}\left(\frac{P}{L}\right)
$$

The blocking force is proportional to the pressure provided and inversely proportional to the actuator length that the volume change to the angle change ratio is a constant. This derivation of the blocking force (Eqs.9-13) is similar to the derivation for the force output of McKibben actuators [31-32].

As implementing Eqs.13 and 14 can be problematic due to the difficulties associated with analytically expressing the volume change as a function of the bending angle or experimentally measuring this variation, we can use Eq.7 described for the whole actuator to estimate the blocking force $F_{B}$. For an experimentally measured radius of curvature $\mathrm{R}$ and experimentally identified flexural rigidity EI, the blocking force $F_{B}$ can be calculated from

$$
\frac{\theta}{L}=\frac{1}{R}=\frac{F_{B} L}{E I}, \quad F_{B}=\theta \frac{E I}{L^{2}}
$$

As the actuators considered in this study have a composite structure, composed of an elastomeric material with nonlinearities, rigid ribs and constraining paper or a bistable thin flexible metal, it is virtually impossible to estimate their effective modulus of elasticity experimentally using the traditional method of tensile testing. Therefore, we have employed Eq.15 to estimate the effective flexural rigidity (EI) of the actuators with and without the bistable thin metal, if the bending angle and blocking force are known, from

$$
E I=L^{2} \frac{F_{B}}{\theta}
$$

We then calculate the area of moment inertia for the cross-sections in Figure 5, and subsequently calculate the effective modulus elasticity of the actuators, as outlined in Section 4.

\subsection{Kinematic Model}

With reference to the bending configurations in Figure 1, the actuator generates a constant curvature with a radius of $\mathrm{R}$ and bending angle of $\theta$. Using the notation in Figure 2, the tip coordinates of the actuator are obtained as 


$$
\begin{gathered}
x=R \sin \theta \\
y=R(1-\cos \theta)
\end{gathered}
$$

\begin{tabular}{|c|c|c|c|c|c|c|c|c|c|c|}
\hline & \multicolumn{10}{|c|}{ Actuator 1: Elastosil, M 4601} \\
\hline $\begin{array}{c}\text { Pressure, } \\
\mathrm{kPa}\end{array}$ & 0 & 10 & 20 & 30 & 40 & 50 & 60 & 70 & 80 & 90 \\
\hline $\begin{array}{l}\text { Angle, } \\
\text { degree }\end{array}$ & 0 & 15.4751 & 33.6811 & 56.4385 & 79.196 & 106.505 & 129.2625 & 165.6744 & 192.9834 & 206.6379 \\
\hline Force, N & 0 & 0.133 & 0.2866 & 0.4506 & 0.6116 & 0.7728 & 0.9663 & 1.1717 & 1.4012 & 1.6767 \\
\hline
\end{tabular}

Table 2: Experimental data used to estimate the flexural rigidity for A1.

\begin{tabular}{|c|c|c|c|c|c|c|c|}
\hline & \multicolumn{7}{|c|}{ Actuator 2: Translucent Silicone } \\
\hline $\begin{array}{c}\text { Pressure, } \\
\mathrm{kPa}\end{array}$ & 0 & 5 & 10 & 15 & 20 & 25 & 30 \\
\hline $\begin{array}{l}\text { Angle, } \\
\text { degree }\end{array}$ & 0 & 13.6545 & 50.0664 & 91.0299 & 127.4419 & 154.7508 & 182.0598 \\
\hline Force, $\mathrm{N}$ & 0 & 0.0795 & 0.1341 & 0.2339 & 0.3087 & 0.3926 & 0.5543 \\
\hline
\end{tabular}

Table 3: Experimental data used to estimate the flexural rigidity for A2.

\section{Experimental Validation of Analytical Models}

The actuator to be tested was placed in front of a grid paper, as shown in Figure 1, with the pressure incremented at either $5 \mathrm{kPa}$ or $10 \mathrm{kPa}$, depending on the type of actuator being tested. The deflection was measured through visual inspection of the angle made between the vertical walls of the tip and the base, as described in Figure 2. The bending angle model in Eq.9 requires the geometric parameters, the area of moment inertia around the neutral axes, the effective modulus of elasticity of the actuators. We have employed Eq.16 to determine the flexural rigidity EI and subsequently the effective modulus of elasticity of the actuators. It must be noted that measuring the modulus of elasticity of the actuator material experimentally will not be accurate as the actuator structure contains a set of ribs in the middle of each chamber and a layer paper to facilitate the bending of the actuators. We conducted blocking force experiments using a high resolution (0.01 g) electronic scale, and the bending angle experiments for two actuators (A1 and A2) made of two different materials. The data in Tables 2 and 3 are used to estimate the effective moduli of elasticity of $386.66 \mathrm{kPa}$ and $169.56 \mathrm{kPa}$ for $\mathrm{A} 1$ and $\mathrm{A} 2$, respectively, containing a piece of constraining paper layer. It must be noted that the initial deflection in the actuators under zero pressure is deducted from each bending angle measurement. We then used the analytical bending model in Eq. 9 to evaluate its validity. The experimental and estimated results are shown in Figures 6a and 7a for A1 and A2, respectively. More experimental results together with the estimated results shown in Figures $6 \mathrm{~b}$ and $7 \mathrm{~b}$ indicate the validity of the bending model and the blocking force model, and the methodology followed to estimate the effective modulus of elasticity of the actuators. The proposed model is a grey box model due to the difficulties associated with incorporating all nonlinear effects in an analytical model, which allows to experimentally identify some parameters of the model using experimental data. This is the due to the fact that the modulus of elasticity of the composite structure is totally different than the bulk modulus of elasticity of the elastomeric materials used to construct the chambers. Therefore, the effective modulus of elasticity of the composite actuator is experimentally identified using the experimental blocking force data. The effective modulus of elasticity was then used in bending angle model to demonstrate the validity of this model. 

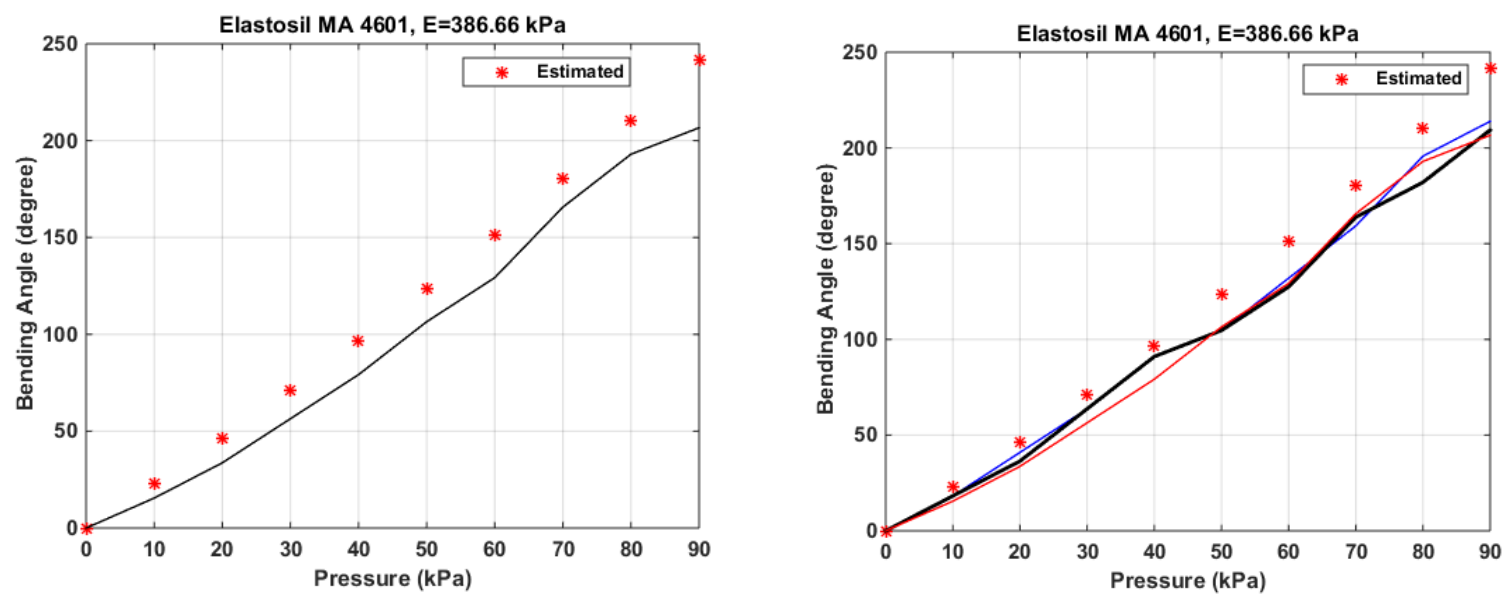

Figure 6: (a) Experimental and estimated results for actuator 1, (b) more experimental results showing the validity of the bending angle and blocking force models. The experimental results are shown with the full line.
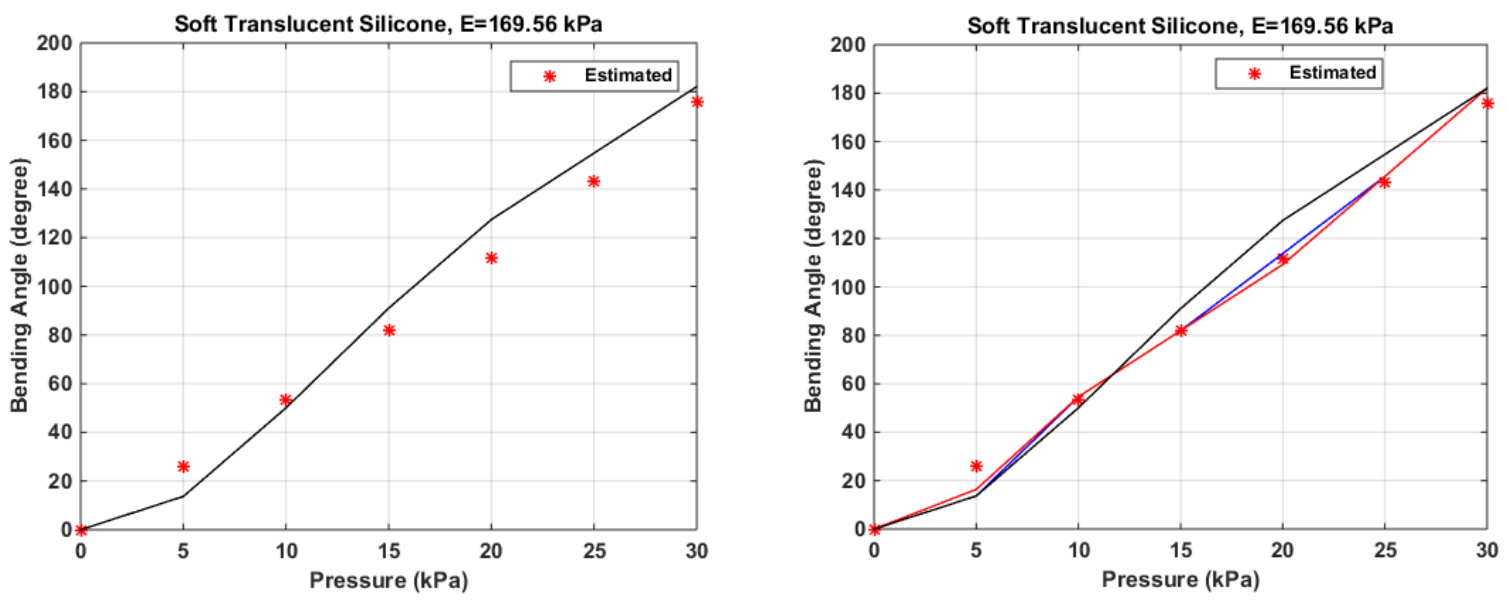

Figure 7: (a) Experimental and estimated results for actuator 2, (b) more results showing the validity of the bending angle and blocking force models. The experimental results are shown with the full line.

\subsection{Experimental results with a bistable thin metal}

As stated before, the soft actuators bend under their own weight. To prevent this, we propose the concept of using a thin bistable metal layer, like a tape measure, to make sure that the actuator keeps its straight configuration in its neutral positon, as shown in the top left corner of Figure 1. We fabricated two actuators containing the tape measure, using the silicone materials. We used the same bending angle and blocking force measurement setups. The results are depicted in Figures 8 and 9 for A1 and A2, respectively. 

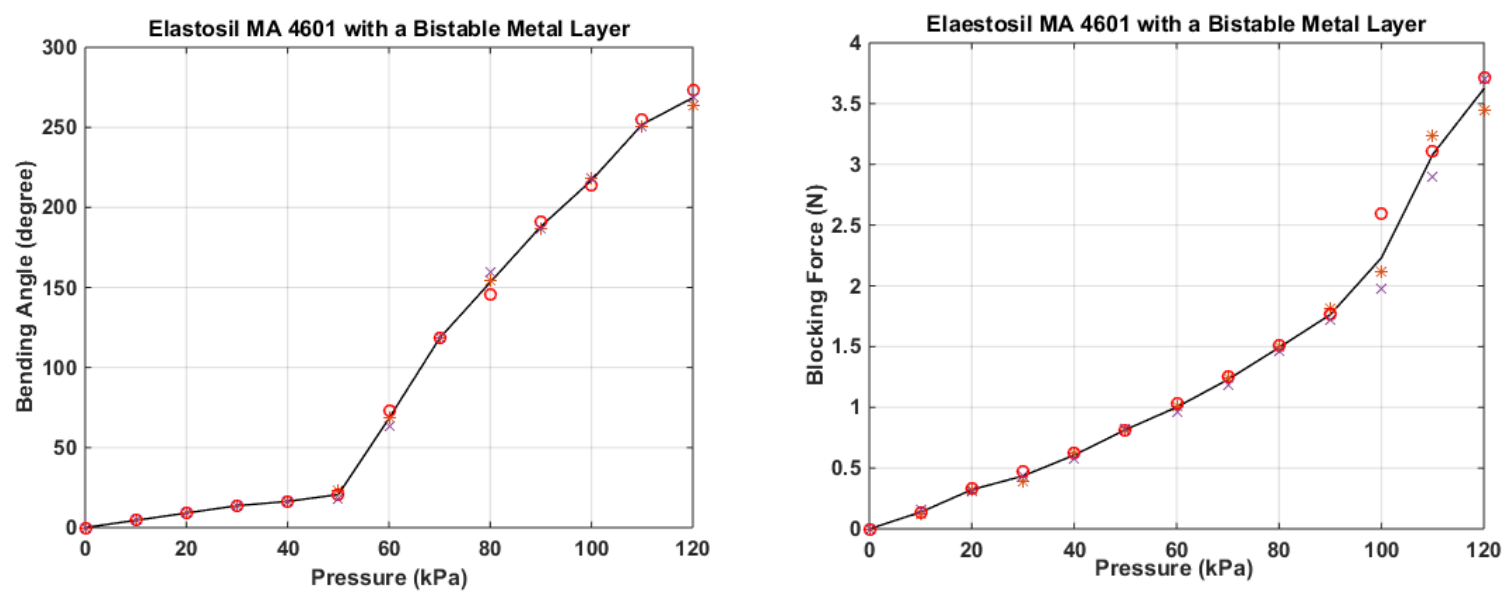

Figure 8: (a) Experimental bending angle versus pressure results, (b) experimental blocking force versus pressure results for A1 with a bistable metal layer consisting of a tape measure. The full line shows the average of the three measurements.

As shown in Figure 8a, the bending angle follows a linear relationship up until $50 \mathrm{kPa}$ at which point the gradient abruptly changes and becomes much higher, continuing linearly until 120 $\mathrm{kPa}$. The gradient before $50 \mathrm{kPa}$ is approximately 0.45 , and the gradient afterwards is 3.65 , increasing by a factor of 8 . The point at which the gradient of the bending angle rapidly increases is the point at which the bistable thin metal changes between its states of stability. Both data sections before and after $50 \mathrm{kPa}$ slightly flatten out at the end of their run. This can be attributed to the bending moment, being highest when the actuator is not vertical, that causes it to slightly bend under its own weight. With reference to the bending model given by Eq.9, when the effective modulus of elasticity is large enough (> $500 \mathrm{kPa}$ ), its nonlinear component can be ignored. This follows that Eq.9 indicates a linear behaviour, as shown in Figure 8a. Using Eq. 16, the effective modulus of elasticity is estimated to be $1.86 \mathrm{MPa}$ and $558.4 \mathrm{kPa}$ for the two states of the thin metal layer for the pressure ranges of $0-50 \mathrm{kPa}$ and $50-120 \mathrm{kPa}$, respectively.
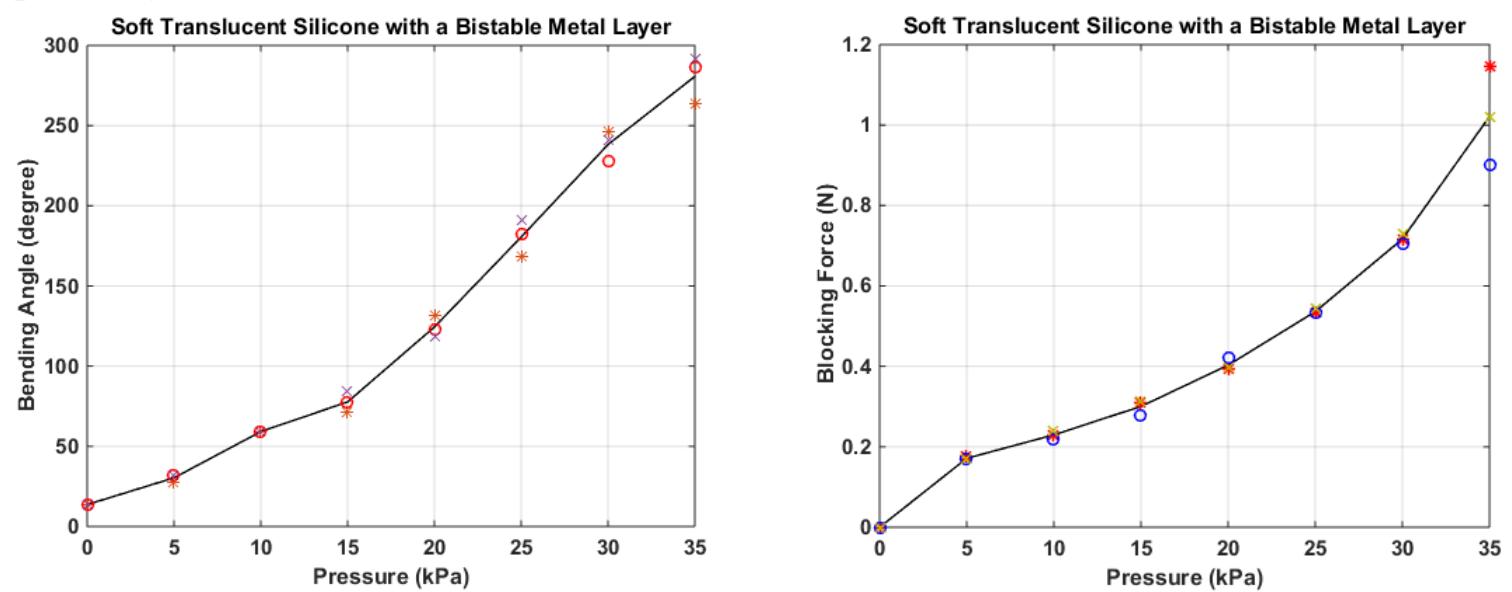

Figure 9: (a) Experimental bending angle versus pressure results, (b) experimental blocking force versus pressure results for A2 with a bistable metal layer consisting of a tape measure. The full line shows the average of the three measurements.

With reference to Figure 8b, the change in the states of the thin metal layer is not indicated up until $90 \mathrm{kPa}$ in the blocking versus-pressure results as the actuator is ideally expected to keep its straight configuration during the force measurements, not allowing significant change in the states of the metal layer. As shown in Figure 8b, the force follows a near perfect linear relationship up until $90 \mathrm{kPa}$. After this point, the gradient increases for the range of 90-120 kPa. 

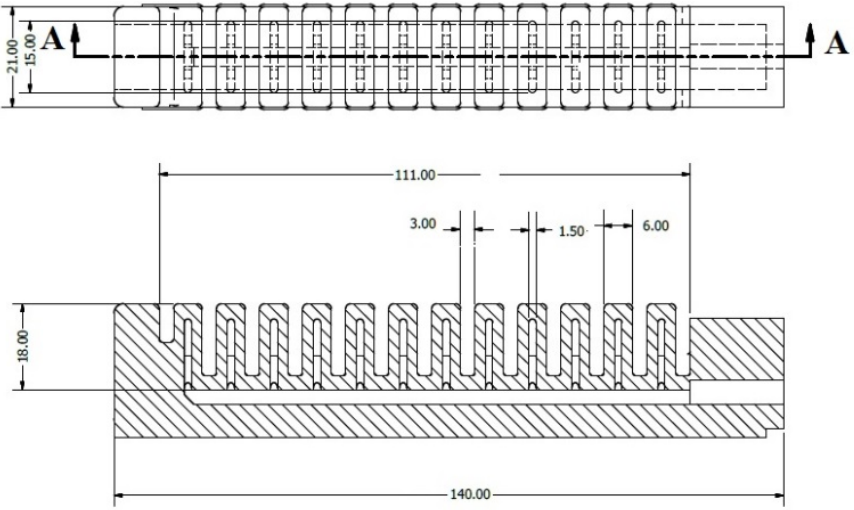

SECTION A-A

(a)
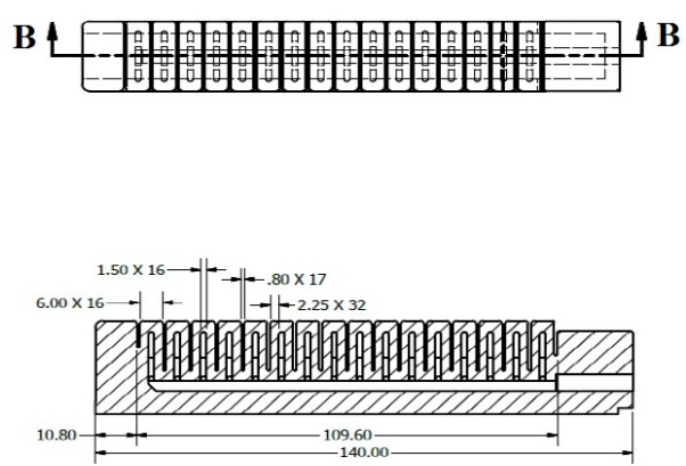

SECTION B-B

(b)

Figure 10: Geometric dimensions of A3 (a) and A1 and A2 (a). It must be noted that the gaps are $3 \mathrm{~mm}$ and $0.8 \mathrm{~mm}$ for A3, and A1 and A2, respectively. The other dimensions are the same.

As presented in Figure 9b, A2 made of the soft translucent silicone has a smaller force output, compared to A1. Therefore, we trimmed (to decrease its width) the bistable thin metal embedded in A2 to decrease its reaction force to bending. The bending angle test for A2 was expected to show the same kind of relationship as A1, but slightly different gradients and points of gradient change. As shown in Figure 9a, the bending angle follows an approximately linear relationship. Although not obvious, at $15 \mathrm{kPa}$ the gradient changes approximately from $4.5 \mathrm{deg} / \mathrm{kPa}$ to $10.4 \mathrm{deg} / \mathrm{kPa}$, increasing approximately by a factor of 2 . The moduli of elasticity for the initial state and final state are estimated as $218 \mathrm{kPa}$ and $156 \mathrm{kPa}$, respectively, using Eq.16. This indicates that the bistable thin metal has changed between its states of stability. This transition occurs at the pressure of $15 \mathrm{kPa}$, whereas for $\mathrm{A} 1$, it occurs at the pressure of $50 \mathrm{kPa}$. The lower critical pressure for A2 can be attributed to two reasons; the softer silicone being used (48 $\mathrm{kPa}$ versus $262 \mathrm{kPa}$ ), and a trimmed (smaller width) bistable thin metal that provides less constraining force to overcome.

The ratio of the gradient change between stability states goes from a factor of 8 for A1 to a factor of 2 for A1. This can be attributed to the net force difference between the bending force of the actuators, and the opposing force of the bistable thin metal being larger for A1. The bistable thin metal in A1 was not trimmed, making it wider and therefore stiffer. As shown in Figure 9b, the blocking force follows an approximately linear relationship up until $30 \mathrm{kPa}$. After this point, the gradient slightly increases. As expected, the softer A2 in Figure $9 \mathrm{~b}$ behaved in a similar manner to that of the stiffer A1 in Figure 8b, with the exception of operating under different pressure ranges. It must be noted that the bending angle versus pressure data in Figure 9a indicate a nonlinear behaviour, as predicted by Eq.9 for an actuator with a relatively smaller modulus of elasticity.

\subsection{Experimental results with an actuator with wider gaps}

We conducted bending and blocking force experiments for an actuator made of Elastosil M4061, which we call A3. The geometric dimensions of this actuator and other two actuators (A1 and A2) are depicted in Figure 10. It must be noted that the gap in A1 and A2, and A3 are $0.8 \mathrm{~mm}$ and $3.0 \mathrm{~mm}$ respectively. As the actuator length is kept the same for all actuators, the number of chambers for A1 and A2, and A3 are 16 and 12, respectively. The experimental results for A3 are shown in Figure 11. These results are used to estimate the effective modulus elasticity of $359 \mathrm{kPa}$, which is slightly below the effective modulus of elasticity $387 \mathrm{kPa}$ for A1 made of the same materials. This difference can be due to the fact that the actuators were fabricated and tested in different days when compared to A1, likely introduced some inevitable measurement and fabrication errors. It must be noted from the results in Figure 11a that the 
actuator with the larger gaps have generated relatively smaller bending angles. One obvious reason is that the number of pneumatic chambers is decreased from 16 to 12, which will decrease the work input causing the bending of the actuator, as described by Eq.10.
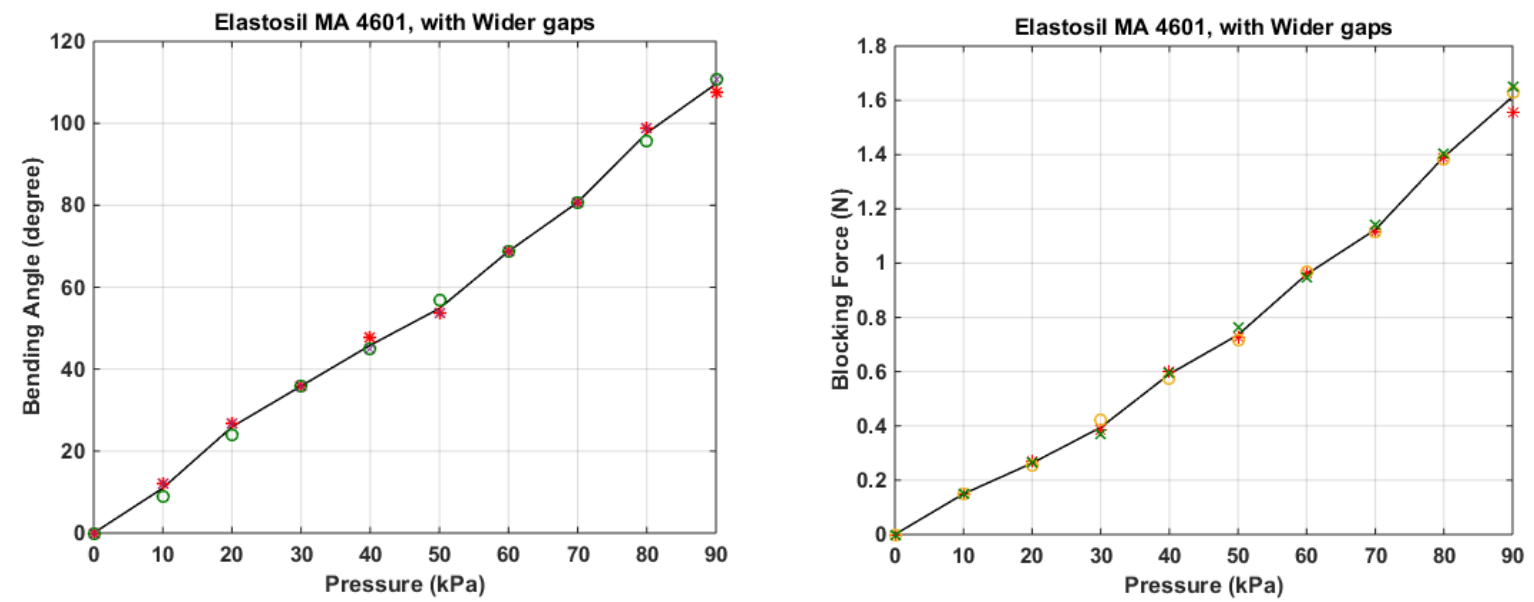

Figure 11: The experimental bending angle versus pressure, and blocking force versus input pressure results for A3, the actuator with wider gaps in the between the chambers.

\section{Applicability: A Soft Robotic Gripper}

A housing was built that could hold three of the actuators made of Elastosil MA 4061 (A1) embedded with the thin metal layer. It was designed to test the feasibility of using the actuators in an industrial gripper. The housing, as shown in Figure 12, was designed to support three actuators equidistant from one another (120 degrees) and offset at an angle of -30 degrees from the horizontal. The entire housing was designed to be attached to a robot manipulator with the housing and actuators acting as the fingers of an effector connected to the tip of the robot manipulator.
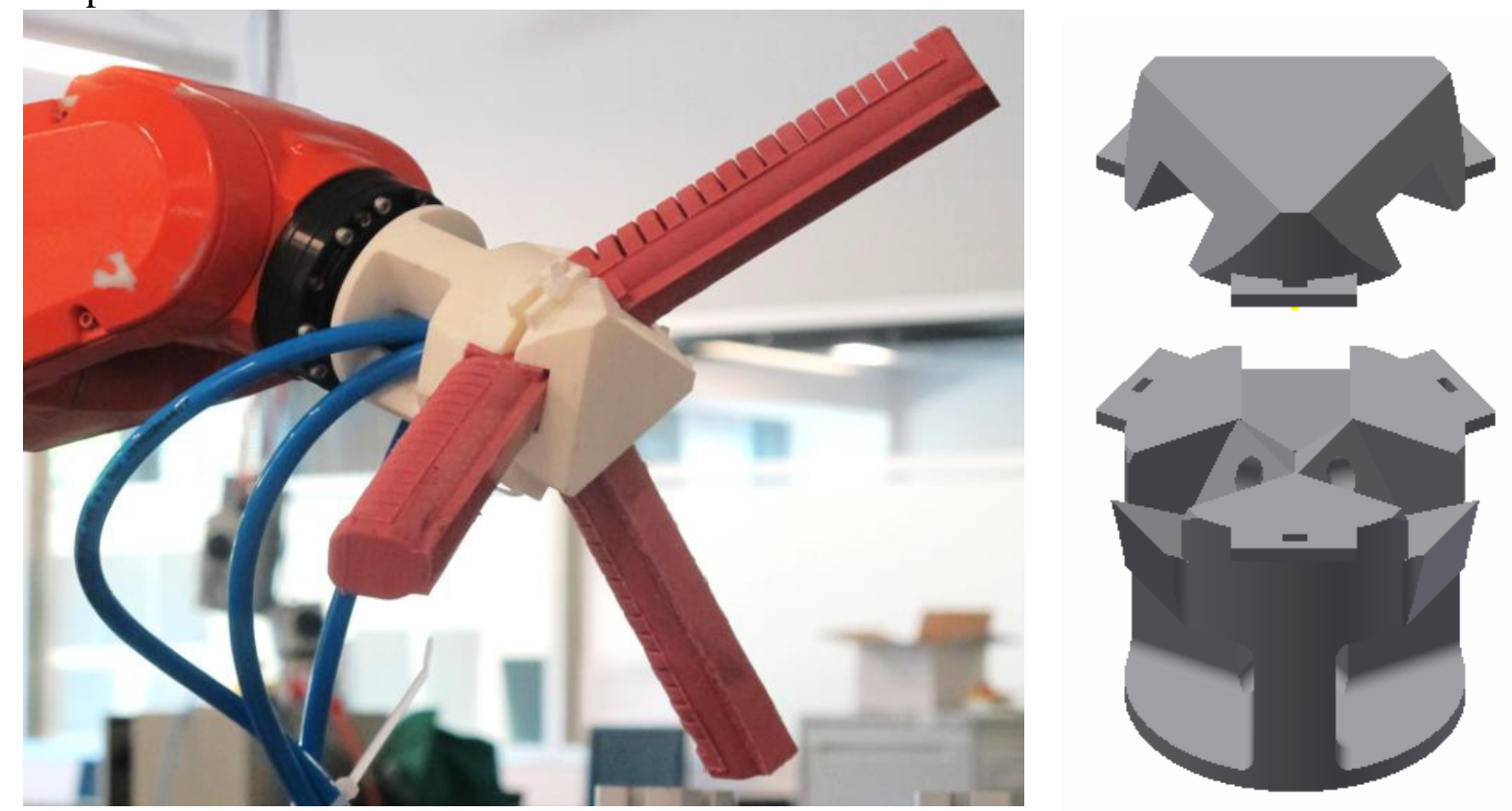

Figure 12: Soft robotic gripper concept: the gripper (left) and housing CAD model (right). It must be noted that each finger is in their straight state- thanks to the bistable thin metal layer providing this.

The gripper's ability was tested with a number of different objects including fruit and vegetables, as illustrated in Figure 13. Due to the length and circular profile of the actuators, the 
gripper was most effective at holding larger and round objects. However, by decreasing the actuator's length and thus its radius of curvature, smaller objects with irregular shapes can be handled with a multi-finger soft gripper. Figure 13 displays the gripper's ability to handle both spherical (a grapefruit) and non- spherical objects (a capsicum). This is also shown in the accompanying video file. The actuator's compliance allows the gripper to adapt to the objects of varying sizes and shapes without requiring any sensory feedback.
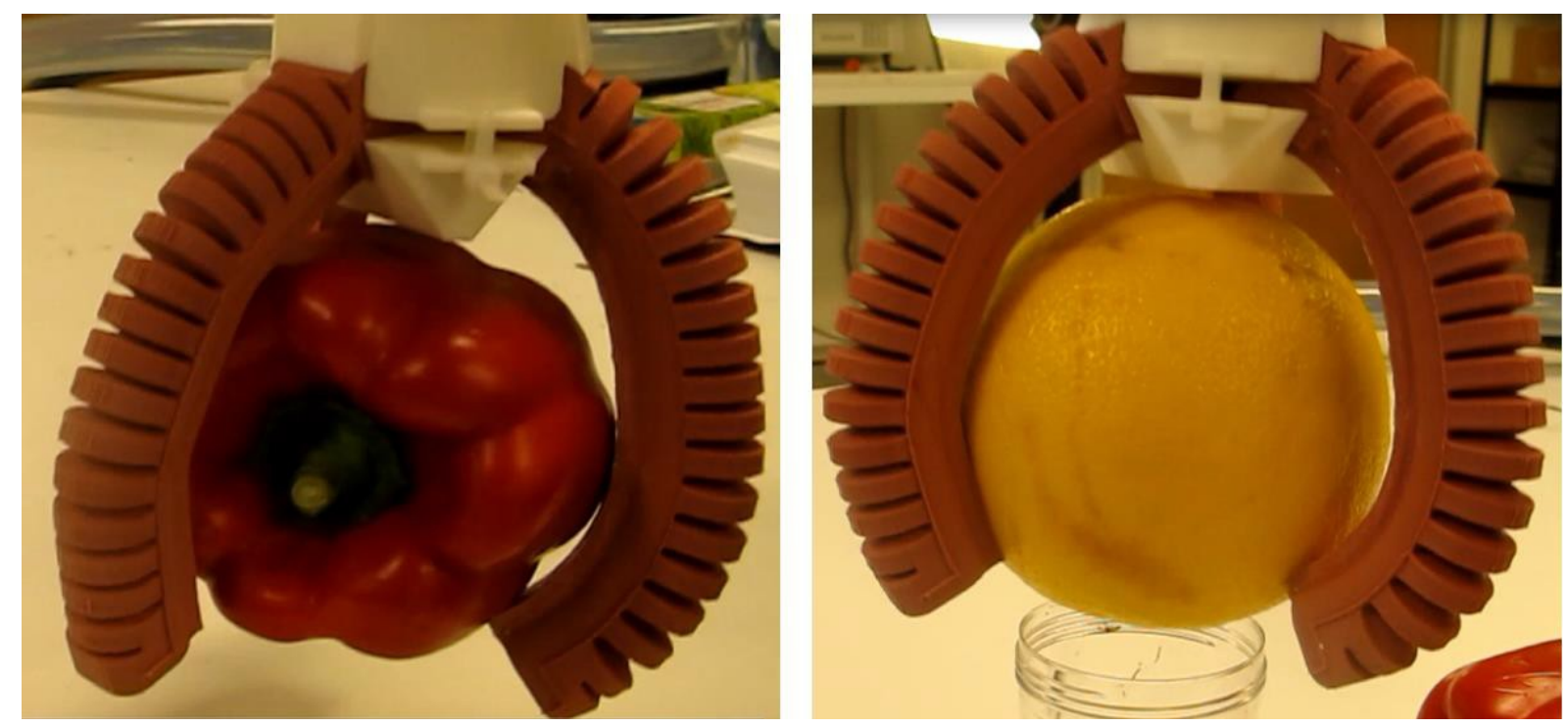

Figure 13: Actuated gripper holding a capsicum (left) and a grapefruit (right).

\section{Conclusions and Future Work}

We have established an analytical model to predict the bending angle of the slender pneumatic actuators made of discrete pneumatic chambers. The actuators were fabricated from two hyperelastic silicones. The analytical model has been validated using the experimental results, While this is the main achievement of this study, we have proposed a simple method to estimate the effective modulus of elasticity of the actuators made of multi-materials with varying material properties. Another important contribution of this study is to propose a thin flexible metal embedded in the topology of the actuators not only to provide the length-restricting effect needed for bending, but also prevent bending of the actuators under their own weight in their neutral states under zero input pressure.

The future work involves optimising the mechanical output of the actuator with different crosssections and materials they are fabricated from. Other than the input pressure, the modulus of elasticity is the parameter significantly affecting the mechanical output of these actuators (as inferred from Eq.9). Taking the modulus elasticity as the important parameter suggests that there is a need for smart materials with a variable modulus of elasticity. Actuators made of such materials can be tailored for various applications as per their variable modulus of elasticity, delivering the proponents of soft robotics in cutting edge applications such as rehabilitation robotics, medical robotics and assistive devices, for which a soft contact is essential to provide compliance matching between the robotic device and its environment [10, 33, 37]. The environment is usually an equally soft body; a human being or agricultural product, requiring sensitive handling. Soft pneumatic actuators could function effectively in these environments by tolerating inaccuracies in the positioning, shape and surfaces of such objects. 


\section{ACKNOWLEDGEMENTS}

ARC Centre of Excellence for Electromaterials (ACES) (Grant No. CE140100012). This work is partly supported by the Intelligent Nano-Tera Research Systems Laboratory at University of Wollongong.

\section{References}

1. D. Rus and M. T. Tolley, "Design, fabrication and control of soft robots," Nature, vol. 521, no. 7553, pp. 467-475, 2015.

2. S. Kim, C. Laschi, and B. Trimmer, "Soft robotics: a bioinspired evolution in robotics", Trends in Biotechnology, vol. 31, issue 5, pp. 287-294, May 2013.

3. S. Bauer, S. Bauer-Gogonea, I. Graz, M. Kaltenbrunner, C. Keplinger, and R. Schwoediauer, "A Soft Future: From Robots and Sensor Skin to Energy Harvesters”, Adv. Mater. 26, 149-162, 2014.

4. G. Alici, Editorial for Special Issue "Mechanics, Control, Design, Conceptualization and Fabrication of Soft Robotic Systems", http://www.mdpi.com/journal/robotics/special_issues/soft_robotic, accessed on May 20, 2016.

5. G. S. Chirikjian and J. W. Burdick, "The kinematics of hyper-redundant robot locomotion”, IEEE Transactions on Robotics and Automation, Vol. 11, no.6 , pp. 781 - 793, December 2005.

6. R. Kang, D. T. Branson, T. Zheng, E. Guglielmino, and D. G. Caldwell, "Design, modeling and control of a pneumatically actuated manipulator inspired by biological continuum structures,” Bioinspiration \& biomimetics, vol. 8, no. 3, p. 036008, 2013.

7. R. J. Webster and B. A. Jones, "Design and kinematic modeling of constant curvature continuum robots: A review," The International Journal of Robotics Research, vol. 29, no. 13, pp. 1661-1683, 2010.

8. R. Pfeifer, M. Lungarella, and F. Iida, "The Challenges ahead for Bio-Inspired Soft Robotics”, Communications of ACM, Vol.55, No.11, pp. 76 - 87, November 2012.

9. H. Hauser, A. J. Ijspeert, R. M. Fuchslin, R. Pfeifer, and W. Mass, "Towards a theoretical foundation for morphological computation with compliant bodies", Biological Cybernetics, vol.105, pp. 355 -370, December 2011.

10. R. Mutlu, G. Alici, M. in het Panhuis, and G.M, Spinks, "3D Printed Flexure Hinges for Soft Monolithic Prosthetic Fingers", Vol.3, No.3, pp.120 -133, September 2016.

11. S. Wakimoto, K. Suzumori, and K. Ogura, "Miniature Pneumatic Curling Rubber Actuator Generating Bidirectional Motion with One Air-Supply Tube”, Advanced Robotics 25 (2011) 1311-1330.

12. P. Polygerinos, Z. Wang, J. T. B. Overvelde, K. C. Galloway, R. J. Wood, K. Bertoldi, and C. J. Walsh, "Modeling of Soft Fiber-Reinforced Bending Actuators", IEEE Transactions on Robotics, Vol. 31, No. 3, June 2015.

13. B. Mosadegh, P. Polygerinos, C. Keplinger, S. Wennstedt, R. F. Shepherd, U. Gupta, et al., "Pneumatic Networks for Soft Robotics that Actuate Rapidly", Advanced Functional Materials, vol. 24, pp. 2163-2170, 2014.

14. F. Ilievski, A. D. Mazzeo, R. F. Shepherd, X. Chen, and G. M. Whitesides, "Soft robotics for chemists," Angewandte Chemie, vol. 123, pp. 1930-1935, 2011.

15. P. Polygerinos, S. Lyne, Z. Wang, L. F. Nicolini, B. Mosadegh, G. M. Whitesides and C. J. Walsh, "Towards a Soft Pneumatic Glove for Hand Rehabilitation”, 2013 IEEE/RSJ International Conference on Intelligent Robots and Systems (IROS), pp. 1512 - 1517, November 3-7, 2013. Tokyo, Japan. 
16. R. F. Shepherd, F. Ilievski, W. Choi, S. A. Morin, A. A. Stokes, A. D. Mazzeo, X. Chen, M. Wang, and G. M. Whitesides, "Multigait soft robot," Proceedings of the National Academy of Sciences, vol. 108, no. 51, pp. 20 400-20 403, 2011.

17. C. D. Onal and D. Rus, "Autonomous undulatory serpentine locomotion utilizing body dynamics of a fluidic soft robot," Bioinspiration \& Biomimetics, vol. 8, no. 2, p. 026003, 2013.

18. M. D. Volder and D. Reynaerts, "Pneumatic and hydraulic microactuators: a review," Journal of Micromechanics and Microengineering, vol. 20, pp. 1-18, 2010.

19. J. C. Yeo, H. K. Yap, W. Xi, Z. Wang, C.-H. Yeow, and C. T.Lim, "Flexible and stretchable strain sensing actuator for wearable soft robotic applications”, Advanced Materials Technologies. Vol.1, DOI: 10.1002/admt.201600018, 2016.

20. R. Deimel and O. Brock, "Novel Type of Compliant and Underactuated Robotic Hand for Dexterous Grasping”, The International Journal of Robotics Research, Vol. 35, No. 1-3, pp. 161--185, 2016.

21. Y. Shapiro, A. Wolf, and K. Gabor, "Bi-bellows: pneumatic bending actuator", Sensors and Actuators A: Physical, Vol.162, pp.484 - 494, 2011.

22. B. Gorissen, W. Vincentie, F Al-Bender, D. Reynaerts, and M. De Volder, "Modelling and bond-free fabrication of flexible fluidic microactuators with a bending motion”, J. of Micromechanics and microengineering, Vol.23, 045012, 2015.

23. B. Chang, A. Chew, N. Naghshineh, and C. Menon, "A spatial bending fluidic actuator: fabrication and quasi-static characteristics”, Smart Materials and Structures, Vol.21, 045008, 2012.

24. Y. Sun, Y. S. Song, and J. Paik, "Characterization of silicone rubber based soft pneumatic actuators”, 2013 IEEE/RSJ International Conference on Intelligent Robots and Systems (IROS), pp. 4446 - 4453, Tokyo, Japan, November 2013.

25. A. D. Marchese, R. K. Katzschmann, D. Rus, “A Recipe for Soft Fluidic Elastomer Robots”, Soft Robots, Vol.2, No.1, pp. 7-25, 2015.

26. G. Alici, "An Effective Modelling Approach to Estimate Nonlinear Bending Behaviour of Cantilever Type Conducting Polymer Actuators", Sensors and Actuators: B. Chemical, Vol.141, No.1, pp. 284 - 292, August 2009.

27. P. Moseley J. Manuel Florez, H. A. Sonar, G. Agarwal, W. Curtin and J. Paik, "Modeling, Design, and Development of Soft Pneumatic Actuators with Finite Element Method”, Advanced Engineering Materials, 18, No. 6. Pp. 978 - 988, 2016.

28. B. S. Homberg, R. K. Katzschmann, M. R. Dogar, and D. Rus, "Haptic identification of objects using a modular soft robotic gripper”, 2015 IEEE/RSJ International Conference on Intelligent Robots and Systems. pp. 1698-1705 Germany, October 2015.

29. R. A. Bilodeau, E. L. White, and R. K. Kramer, "Monolithic fabrication of sensors and actuators in a soft robotic gripper,", 2015 IEEE/RSJ International Conference on Intelligent Robots and Systems, pp. 2324-2329, Germany, October 2015.

30. D. I. Bower, “An Introduction to Polymer Physics”, Cambridge University Press, pp. 170-172, 2002.

31. B. Tondu, "Modelling of the McKibben artificial muscle: A review", Journal of Intelligent Material Systems and Structures, Vol. 23, No.3, pp. 225-253, 2012.

32. C. P. Chou and B. Hannaford, "Measurement and modelling of McKibben pneumatic artificial muscles", IEEE Transactions on Robotics and Automation, Vol.12, No.1, pp.90-102, 1996.

33. R. Mutlu, S. K. Yildiz, and G. Alici, M. in het Panhuis, and G.M, Spinks, "Mechanical Stiffness Augmentation of a 3D Printed Soft Prosthetic Finger", 2016 IEEE/ASME 
International Conference on Advanced Intelligent Mechatronics, pp.7-12, Banff, Canada, July 2016.

34. D. Yang, B. Mosadegh , A. Ainla , B. Lee , F. Khashai , Z. Suo , K. Bertoldi , and G. M. Whitesides, "Buckling of Elastomeric Beams Enables Actuation of Soft Machines, Advanced Materials, Vol.27, 6323-6327, 2015.

35. Y. Hwang, O.H. Paydar, R.N. Candler, "Pneumatic microfinger with balloon fins for linear motion using 3D printed molds”, Sensors and Actuators a-Physical 234 (2015) 65-71.

36. H.K. Yap, H.Y. Ng, C.-H. Yeow, "High-Force Soft Printable Pneumatics for Soft Robotic Applications”, Soft Robotics 3(3) (2016) 144-158.

37. G. Agarwal, N. Besuchet, B. Audergon and J. Paik, "Stretchable Materials for Robust Soft Actuators towards Assistive Wearable Devices”, Sci. Rep. 6, 34224; doi: 10.1038/srep34224 (2016).

38. S. Timoshenko, "Strength of Materials, Part I, Elementary Theory and Problems”, 2nd edn., D. Van Nostrand Company (1940). 